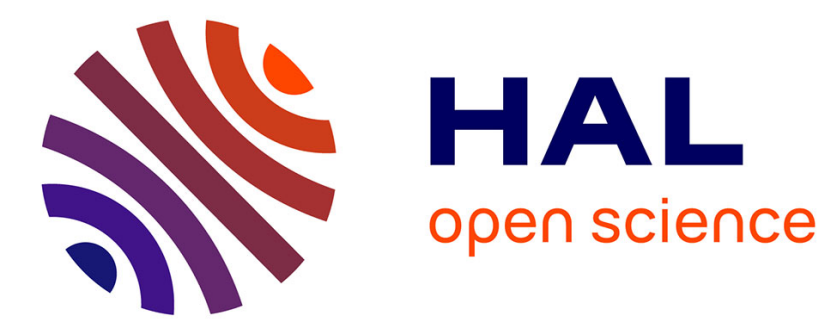

\title{
A rationale of digital documentary editions
}

Elena Pierazzo

\section{To cite this version:}

Elena Pierazzo. A rationale of digital documentary editions. 2011. hal-01182169

\section{HAL Id: hal-01182169 \\ https://hal.univ-grenoble-alpes.fr/hal-01182169}

Preprint submitted on 30 Jul 2015

HAL is a multi-disciplinary open access archive for the deposit and dissemination of scientific research documents, whether they are published or not. The documents may come from teaching and research institutions in France or abroad, or from public or private research centers.
L'archive ouverte pluridisciplinaire HAL, est destinée au dépôt et à la diffusion de documents scientifiques de niveau recherche, publiés ou non, émanant des établissements d'enseignement et de recherche français ou étrangers, des laboratoires publics ou privés.

$$
\text { Copyright }
$$




\section{A Rationale of Digital Documentary}

\section{Editions}

\section{Elena Pierazzo}

Department of Digital Humanities, King's College London, UK

\section{Abstract}

Publishing the diplomatic edition of a document on the web instead of in print implies a series of methodological and practical changes in the nature of the published text and in the operations to be performed by the editors. For print the choice of which features to include in the transcription is limited largely by the limits of the publishing technology. In contrast, the digital medium has proved to be much more permissive and so editors need new scholarly guidelines to establish "where to stop". This article discusses a list of criteria and parameters for choosing which features to include in transcriptions. It also sketches the theoretical implications which result from the change of medium and technology. It is argued that the very definition of "diplomatic edition" needs to be substantially revised if the edition is published on the web. Even more importantly, the discussion argues for the existence of a new editorial object which is generated by the changed conditions: a new publication form called the "digital documentary edition" which is composed of the source, the outputs and the tools able to produce and display them. 
The preparation and publication on the Web of digital scholarly editions, especially those based on transcriptions of manuscripts, are at the centre of lively debate among scholars. In particular, scholars have tried to answer to the following questions: are digital editions different from printed ones? If so, in which ways? Do they represent an advancement of textual scholarship or just a translation of the same scholarship into a new medium? ${ }^{1}$ It is the argument of this article that editions as we know them from print culture are substantially different from the ones we find in a digital medium. They may perhaps present the material in the same way as their printed counterpart, as lamented by Robinson (2002), but they are intrinsically different with respect to traditional editorial models, especially those editions encoded according to the Guidelines of the Text Encoding Initiative (TEI).

The discussion that follows ${ }^{2}$ will try to explain the reasons behind this statement. The argument will use as a case study the Jane Austen's Fiction Manuscripts Digital Edition, a three-year AHRC-funded project devoted to the transcription and publication of Jane Austen's holographic fictional manuscripts. ${ }^{3}$ The discussion will focus on some of the theoretical and practical implications of preparing a diplomatic edition of the manuscripts and presenting them side-by-side with their digital facsimiles.

\section{Definitions and Theoretical Framework}

\subsection{What is a Diplomatic Edition?}

According to its classic definition, a diplomatic edition comprises a transcription that reproduces as many characteristics of the transcribed document (the diploma) as allowed by the characters used in modern print. ${ }^{4}$ It includes features like line breaks, page breaks, abbreviations and differentiated letter shapes. Recently, for modern manuscripts (especially draft manuscripts) a new form of diplomatic edition has been developed which attempts to 
reproduce also the topographic collocation of different writings, including functional signs and marks, again within the limits of the print medium. This new type of edition is called super-diplomatic or type-facsimile. Diplomatic editions are very expensive, in terms of both production and, especially, publication. In the print world, they have been used mainly for documents that are either very short, very important, or both, or where a facsimile reproduction was impossible or infeasible, for example because of cost. The diplomatic edition therefore has been, and still is, considered as a sort of surrogate of a facsimile edition.

In a digital environment, the cost of publication (though not necessarily that of production) has been remarkably reduced, and therefore an increasing number of such editions are now being published on the web. This fact, combined with the greater possibilities for representation which the digital medium allows, has raised a lot of interest in this type of edition and has generated several discussions concerning the methodological and pragmatic problems that have arisen.

Before going further, however, and at the risk of stating the obvious, it is worth first discussing a question of terminology which has substantial implications: namely whether a diplomatic edition is the same as a transcription. As already discovered by Huitfeldt and Sperberg-McQueen (2008, p. 296) the term transcription has been used to denote both an act (the act of transcribing) and also "the product of that act, that is, a document", or even "the relationship between documents - one document may be said to be a transcription of another document". This ambiguity has lead to some confusion in the literature. Meulen and Tanselle have used the word transcription in opposition to critical text as possible parts of an edition (1999, p. 203). Similarly Gabler seems to imply that transcriptions and diplomatic editions are the same thing, speaking as he does of "diplomatic transcription" (2007, p. 204). In contrast, it is argued here that the terms transcription and diplomatic edition identify two very 
different objects: one a derivative document that holds a relationship with the transcribed document, and the other a formal (public) presentation of such a derivative document. The editor will first transcribe a primary source, thereby creating a transcription; this transcription will be corrected, proofread, annotated and then prepared for publication. Once published, this new object will become a diplomatic edition. The two products will possibly contain the same text, but while the first will be a private product, the latter will be a publicly published one. These two objects therefore represent two different stages of the same editorial process, although the first can exist without the second. The following discussion will evaluate both these stages in turn.

\subsection{What Does a Diplomatic Edition Contain?}

Michael Sperberg-McQueen declares that there are three things to consider when we transcribe a text:

1. There is an infinite set of facts related to the work being edited.

2. Any edition records a selection from the observable and the recoverable portions of this infinite set of facts.

3. Each edition provides some specific presentation of its selection (2009, p. 31, italics original).

His assertion (which is quite correct) that any edition records only a selection of the facts has two important logical consequences for transcriptions and diplomatic editions. First, no transcription, however accurate, will ever be able to represent entirely (i.e. faithfully) the source document. Some characteristics of the manuscript are irredeemably lost by transcribing it, for instance the variable shape and spacing of handwritten glyphs versus the constant shape of digital fonts or typescripts. As Hans Walter Gabler says, "clearly the diplomatic transcription is already a distinct abstraction from the document" 
(2007, p. 204). This fact is also clearly recognized by Meulen and Tanselle: “Obviously a transcription cannot exactly reproduce the relative precision or carelessness with which handwritten letters are formed, or their relative sizes, or the amount of space between words and lines" (1999, p. 201). This point is worth emphasizing for reasons that will be clear shortly. Second, if every editor necessarily selects from an infinite set of facts, it is evident that any transcription represents an interpretation and not a mechanically complete record of what is on the page. The process of selection is inevitably an interpretative act: what we choose to represent and what we do not depends either on the particular vision that we have of a particular manuscript or on practical constraints. As Huitfeldt and Sperberg-McQueen argued, "the concept of transcription largely consists in a systematic program of selective alteration, coupled with selective preservation of information" (2008, p. 302). This was written as a response to a declaration by Meulen and Tanselle in which those authors affirm that the concept of transcription cannot be associated with one of alteration:

numerous discussions of the subject, including some of the most influential, allow for making certain classes of alteration when transcribing manuscript texts, as if a conscious program of alteration is compatible with the concept of transcription (1999, pp. 201-202).

However this statement contradicts a passage that immediately precedes it, in which the role of the transcriber (editor) in making informed choices and decisions is brought to light:

Judgment is necessarily involved in deciding what is in fact present [in the manuscript], as when an ambiguously formed character resembles two different letters; but the transcriber's goal is to make an informed decision about what is actually inscribed at each point (Meulen and Tanselle, 1999, p. 201).

This leads in turn to the canonical distinction between record and interpretation made by Hans Zeller, a distinction which has deeply influenced contemporary German editorial 
theory (Zeller, 1995 and in general Gabler et al., 1995) and which clearly distinguishes what is on the page (Befund) from the interpretation of this phenomenon (Deutung). It is the opinion of this author that this distinction has proven to be largely artificial. As argued elsewhere, selection at least is an interpretative act from which alterations inevitably spring. What is the nature of those alterations performed as a direct consequence of any editorial act? Huitfeldt and Sperberg-McQueen seem to consider such alterations to be substitution (2008, p. 302). On the other hand, Robinson and Solopova have argued that editorial alterations are acts of translation and not of substitution:

transcription of a primary textual source cannot be regarded as an act of substitution, but as a series of acts of translation from one semiotic system (that of the primary source) to another semiotic system (that of the computer). Like all acts of translation, it must be seen as fundamentally incomplete and fundamentally interpretative (Robinson and Solopova, 1993, p. 21).

Most probably the authors of the aforementioned articles agree on the substance, if not on the terminology. But, whatever the nature of the relationship between exemplar and transcript, it is clear that the alterations which lead from the former to the latter are interpretative and irreversible: two scholars, given the same transcriptional criteria, are most likely not to produce the same transcription of the same exemplar; furthermore it is impossible to use a transcription to reconstruct the exact substance and appearance of the exemplar, assuming that one can tell them apart. The alterations performed by the editor are only partially accountable and documentable.

If a transcription consists of "a systematic program of selective alteration" (Huitfeldt and Sperberg-McQueen, 2008, p. 302) and is a substantially interpretative act, is it then possible to maintain some level of objectivity in performing such operations? According to Claus Huitfeldt the answer seems to be yes: "Thus we may conclude that there is such a thing as objectivity of interpretation: the vast majority of decisions we make 
in this realm are decisions on which all (or most) competent readers agree or seem likely to agree" (2006, p. 196). This conclusion comes after an affirmation that representation and interpretation ("the subjective and the objective") are not a dichotomy but the two extremes of a continuum. The argument of social agreement seems to be supported by Peter Robinson when he wrote that ' An ' $i$ ' is not an ' $i$ ' because it is a stroke with a dot over it. An ' $i$ ' is an ' $\mathrm{i}$ ' because we all agree that it is an ' $i$ '” (Robinson, 2009, p. 44). These scholars seem then to imply that objectivity is defined exclusively by mutual agreement: if scholars as competent readers agree on something, then by this definition that thing is objective. However, this definition, and particularly its implied emphasis on scholarly opinion, is at odds with the received definition of the word objectivity: "the ability to consider or represent facts, information, etc., without being influenced by personal feelings or opinions" (OED). In fact (and, to be fair, this is present more or less implicitly in the argument of both Huitfeldt and Robinson) the transcription of what is on the page is perceived by Huitfeldt as "less objective and relievable" than that which can be measured or handled mechanically (2006, p.195). Perhaps then we should simply say that the notion of objectivity is not very productive or helpful in the case of transcription and subsequently of diplomatic editions and that we should instead make peace with the fact that we are simply doing our work as scholars when transcribing and preparing a diplomatic edition. In place of the unachievable universal truth of objectivity it is argued here for informed, circumspect, documented, scholarly interpretation. We should therefore conclude that a diplomatic edition, like any other edition, is an interpretative, scholarly product, based on the selection of features transcribed from a specific primary source. 


\section{Selection as Scholarly Activity: Where to Stop}

Scholarly choices constitute the base of any transcription and subsequent diplomatic edition. But that then raises the question how to choose. Which features of the primary source are we to reproduce in order to be sure that we are following "best practice"? Are there any shared guidelines to inform our choices? Where shall we stop? According to Tanselle "the editor's goal is to reproduce in print as many of the characteristics of the document as he can" (1978, p. 51). This statement is of very little help in a digital context where the medium is so much less limiting, as clearly declared by Driscoll while commenting on the possibilities offered to those encoding with TEI: "to all intents and purposes there is no limit to the information one can add to a text - apart, that is, from the limits of the imagination" (2006, p. 261). Forcing Driscoll's words a little, we might conclude that one possible and tempting answer to the question "where to stop" could be "nowhere", as there are potentially infinite sets of facts to be recorded. Nevertheless such an answer opens the field to more theoretical and practical concerns.

When Sperberg-McQueen states that an edition records a selection of facts, he implies that a transcription/diplomatic edition behaves as a model of the physical object (the manuscript). ${ }^{6}$ If we pursue this line then, if there is an infinite set of facts to be observed within the physical object, "no limits" might lead us to create a model which aspires to equal the object to be studied. But a model must be simpler than the object it models, otherwise it will be unusable for any practical purpose. This is a fact that has been demonstrated by the "fathers" of the theory of models:

Let the model approach asymptotically the complexity of the original situation. It will tend to become identical with that original system. As a limit it will become that system itself. [...] Lewis Carroll fully expressed this notion in an episode in Sylvie and Bruno, when he showed that the only completely satisfactory map to scale of a given country was that country itself. (Rosenblueth and Wiener, 1945, p. 320). 
So, we must have limits, and limits represent the boundaries within which the hermeneutic process can develop. The challenge is therefore to select those limits that allow a model which is adequate to the scholarly purpose for which it has been created.

In print, it appears that the limits are set by what can be represented by the typographic characters and by the printing house, as declared by Meulen and Tanselle: "by 'transcription' we mean the effort to report - insofar as typography allows - precisely what the textual inscription of a manuscript consists of' (1999, p. 201). In the digital environment, however, the possibilities of the web extend far beyond the recoding of the features recommended by Tanselle such as punctuation, spelling, letter shape (long s, i-j, u$\mathrm{v}$, for instance), capitalisation, abbreviations, authorial errors, deleted readings, and added texts (maintaining or marking their positioning) (1978, pp. 50-51); one can compare this list with the so-called ultra diplomatic editions which integrate transcription and facsimile as found in Gabler's The Primacy of Document in Editing (2007, p. 205; compare also Elsschot, 2007). The Austen Digital Edition itself offers sophisticated ways of presenting the interlinear insertions, and superimposed and displaced corrections which exemplify some of the options offered by the web. It is clear that we have just started to test the limits of the digital environment and so we cannot use these limits of the medium as a pragmatic answer to the question "where to stop". In addition, to do this will also be allowing the technology to drive scholarly choices, something Michael Hunter warns against when he remarks that what is possible is not necessarily useful:

An electronic edition is like an iceberg, with far more data potentially available than is actually visible on the screen, and this is at the same time a great opportunity and a temptation to overdo things. When so many possibilities exist, there is a danger of technological considerations of what can be done taking priority over intellectual considerations of what is actually desirable or necessary in any particular case (Hunter, 2007, p. 71). 
The limits established by print technology have too often offered an easy answer to the question where to stop: in fact, the best practice recommendations for printed editions could equally qualify as the kind of technology-based decisions which Hunter warned against (Tanselle 1978, Meulen and Tanselle 1999). It is only with the advent of digital editions that we have started to understand that what we need is scholarly guidance on our decision making process, that we need to rethink the reasons why we make our transcriptions, and that this approach should apply to print and digital editions alike.

\subsection{A Grid of Features}

While transcribing, while translating (or substituting) the symbols in the manuscript page into the graphemes that are available from the tool the editor is using for transcription, informed choices need to be made on what to include because it is relevant and what can be safely omitted. In order to make these choices, we must understand which features are present in the exemplar. Let us therefore try to list the most common features one might want to consider from the infinite set of facts that can be found on the written page:

- Documentary features: dimensions, inks, tears, alterations to the integrity of the physical object.

- Topology: structure and layout of the document, collocation of writings and other features in the writing surface.

- Handwriting: number of hands, letter shapes, ligatures.

- Orthography: spelling, diacritics.

- Writing features: which can be split into:

○ Reading facilitators: capitalization, punctuation, spacing.

○ Shorthands: abbreviations, symbols, cyphers. 
- Genesis: revisions, deletions, additions, functional marks and other evidence about how the content of the document was produced.

- Textuality: paragraphs, headings, verses, tables, lists, rubrics and other structural divisions.

- Semantics: dates, names of people, of places, keywords.

- Linguistics: part of speech, lemmatization, syntax.

- Decorations and other graphical components: miniatures, drawings, doodles.

- Others: infinite.

While some of these parameters correspond to graphic evidence on the writing surface (letter shapes, ligatures, graphic components), others represent meta-information, such as dimensions, or qualifications of words in terms of both semantics and grammatical functions. While the former group of features can be represented with more or less any system of transcription, the latter can be recorded only by using some types of writing and editing system: for instance an XML (TEI) based system will satisfy this requirement, while a plain or unstructured text format will hardly be fit for the job. In other words we need a system that allows the shift from the mimetic to the analytic level of the editing process. It is important to note that while many of the previous considerations could be applied to any kind of digital transcription, the ones that follow assume that the technology chosen by the editor is some sort of markup language or a technology that allows the recording of metainformation in the way described above, possibly following the TEI Guidelines. The act of transcribing will therefore now be considered together with the act of encoding the transcribed information. It is beyond the scope of this article to investigate conceptual transformation and the level of computational literacy required of the editor when she or he becomes an encoder (a recent discussion of which can be found in Sutherland and 
Pierazzo 2011); it must be sufficient here to mention that this is not necessarily a smooth or painless evolution and that it comports all sorts of theoretical and practical consequences (Vanhoutte 2000, Rehbein 2010, McLouglin 2010).

When starting a transcription for the purpose of providing a digital diplomatic edition, the features listed above need to be considered one by one for inclusion in one's criteria. In this analysis decisions are taken according to five parameters:

1. The purpose of the edition (or the needs of the editors);

2. The needs of the others (prospected readers, scholars);

3. The nature of the document;

4. The capabilities of the publishing technology;

5. The costs of encoding/the amount of time available for the job.

Features and parameters can then be then organized into a table as follows:

Table 1 - Grid of Features

\begin{tabular}{|l|l|l|l|l|l|}
\hline & Purpose & Readers & Nature of & Publishing & Costs \\
\hline Document & & & Document & Technology & \\
\hline Topology & & & & & \\
\hline Handwriting & & & & & \\
\hline Orthography & & & & & \\
\hline Reading & & & & & \\
\hline Facilitators & & & & & \\
\hline Shorthands & & & & & \\
\hline
\end{tabular}




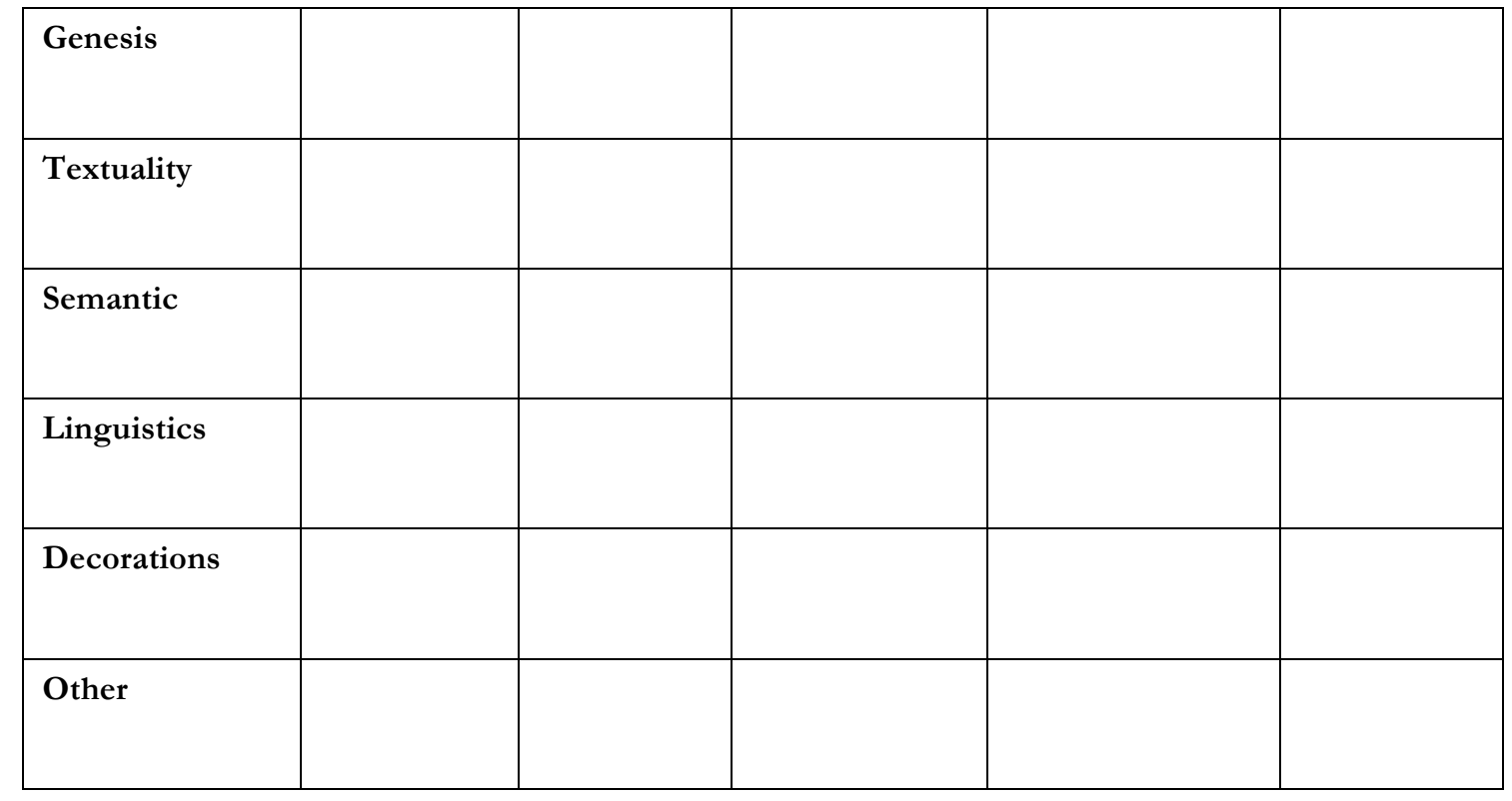

These parameters will be now discussed in turn.

\subsection{Costs}

The examination of the parameters will start with the last one, as it is the one normally considered the fundamental discriminating criterion, namely the cost of encoding and the time available for the job. The following statement from Marilyn Deegan may exemplify this general attitude (words which are strikingly reminiscent of Driscoll's [2006] with their reference to the imagination as the ultimate limit for digital editions): "In this new world, the limits are not what will fit on the page or between covers. The new constraints are time and money; with a sufficiency of both, the technology will allow us to go as far as our imagination leads" (Deegan, 2006, p. 361).

We all know how important economic considerations are in our decision-making processes; almost all of our research projects are funded for a specific time-span and budget, and so it is fundamental to ensure that the transcription (and encoding) is feasible within this lifetime. The decision whether or not to encode a specific feature will be heavily 
determined by the cost of encoding it, and it would be naive not to admit this. Economic considerations may then be used as the pragmatic limits of the level of transcription we are looking for, in the same way that the limits of the typography worked for print-based publications; indeed this parameter enforced some choices for the Austen Digital Edition, as will be mentioned in the following sections. On the other hand one may well argue that such decisions should be scholarly rather than economic, and, even within the limitations of budget, guidance on best practice is desirable to ensure that the money is well spent.

\subsection{The Purpose of the Edition, or the Needs of the Editors}

According to Robinson, "decisions about what one should encode and should not encode are to be determined according the purpose of our encoding” (2009, p. 46): this is certainly true, but it needs to be refined a little to be really useful. To achieve the purpose of the edition and meet the editors' needs, one needs to ask which features bear a cognitive value, that is, which are relevant from a scholarly point of view.

In order to exemplify this point some of the editorial practices of the Jane Austen's Fiction Manuscripts Digital Edition will be presented. ${ }^{7}$ The purpose of the project was to offer a reliable scholarly diplomatic transcription of the surviving autograph manuscripts, together with high quality facsimiles of the same manuscripts, with the aim of favouring the study of the genetic process of such manuscripts along with the linguistic and orthographic habits of the author. The manuscripts constituting this corpus can be classified as working drafts and fair copies, at least some of which were also confidential publications, that is, shared among a group of readers.

\subsubsection{Topological Description}

Page and line breaks have been preserved as well as other layout features such as right or centre alignments of paragraphs, headings, and other blocks of texts, limited to a certain 
amount of precision. In some of the fair copies in particular the layout was carefully planned by the author with the apparent intention of imitating printed books, and so such features have been considered essential characteristics of the documents. It is worth mentioning that this and some of the other features that are explained further below have not been included just because they happened to be "on the page", but because they could possibly give an insight into a particular aspect of Austen's manuscript (work) production which had not hitherto been studied.

\subsubsection{Handwriting and Letter Shapes}

Normal s and long s ( $\int$ have been distinguished. Austen's handwriting is surprisingly little studied (if at all) and this allowed to obtain statistical measures about her writing habits.

\subsubsection{Spelling and Diacritics}

Austen's favoured spellings have been preserved and marked, such as the inversion of the diphthong ie into $e i$ (as in friend spelt freind). Her different methods for marking word breaks have also been preserved, and the different lengths of her dashes, in order to measure writing habits, consistency, and rate of variation within and amongst manuscripts, types of manuscript, and time-periods. The decision was made to record three different lengths of dash: short, medium and long. These lengths have been measured in an approximate and relative way; relative in that the length was considered only compared to others within the given manuscript. With a corpus of documents ranging from when Austen's teenager years to her death, and with gaps of decades between some documents, it was not considered meaningful to compare the absolute length of (say) a short dash in the Juvenilia with that of her last working manuscripts. The decision to measure the length approximately was driven by the consideration that the author was not using a ruler while writing; hence, if she 
did indeed attribute any meaning to the length of dashes, she must have been able to appreciate it by sight.

\subsubsection{Capitalization and Punctuation}

Capital letters were preserved and marked; Austen used these inconsistently for any part of speech, so we have distinguished nouns, verbs, pronouns, adjectives, articles, and adverbs. The original fluctuating punctuation was also kept.

\subsubsection{What Was Not Encoded}

What was left behind or represented loosely is the exact location of the interlinear insertions. Handwriting does not have regular spacing nor regular dimension, therefore, once digitized, the reproduction of its relative and absolute positioning on the screen is very difficult to achieve. The coordinates of every letter could have been specified but it has been thought unreasonable and ultimately beyond the scope of the edition for at least two reasons: first, it would have given very little insight into Austen's way of working and, second, it would have been enormously time consuming. In fact, it has been considered that to understand the way Austen worked (which is one of the purposes of the edition) it was enough to represent insertions positioned in the interlinear space only approximately: the availability of the facsimile would do the rest.

Linguistic features have not been encoded, either from a semantic or a grammatical point of view, except that the part of speech for capitalized words other than nouns was indicated. Similarly, any "proper" genetic markup was not included: while this was a desirable outcome of the project and fitted the purpose of the edition, it could not be managed in the time available for the job. Furthermore, genetic markup was not yet available in TEI at the time of the project: a group had been formed for this purpose, 
collecting the needs of the Austen Digital Edition together with many others, but the results arrived too late to be included in the present project phase. ${ }^{8}$

\subsection{The Needs of the Others}

From the editors' interpretation of the text and of the author(s) intentions it is necessary now to consider the readers of the edition and ask what must be added to the edition itself to satisfy their needs and expectations. Arguably some of the choices made by the editors can be motivated both by the purpose of the edition and by the needs of the readers, and one might also argue that making an accessible edition is one purpose of the edition.

Nevertheless the distinction is still useful, based as it is on editorial interpretation on the one hand ('the purpose of the edition') and what it takes to make such interpretation clearly intelligible on the other ('the needs of the others').

The potential users of a digital edition can be grouped into two categories:

1. The intended target (scholars, students, probably of the same discipline as the editors).

2. The Others, which can be split in two further categories:

- Scholars of other disciplines who may approach the edition for purposes which we cannot foresee.

- Generic readers.

The problem of the two audiences (readers who are scholars of the same discipline and readers who are not) has been well described by John Lavagnino (2009); it is not a new problem, but only with digital publication have editors started to face it in a more substantial way. While books are standard devices the function of which is more or less always the same (but again see Lavagnino, 2009), websites are very different from each other, and the fact that users can use (i.e. find the information in) one does not imply that they can use any other. Therefore website creators and, among them, digital editors must 
consider the needs of the users when preparing their publication and, in consequence, considerations about what kind of users to expect and how to meet their needs have become a common concern for any kind of digital publication.

Two objects in particular have been outlined as the most representative embodiment of the inaccessibility of the traditional (printed) scholarship: the critical apparatus and genetic editions, including diplomatic transcriptions.' A way out from the obscurities of transcriptions of modern manuscripts (the "confusion of brackets": Hunter, $2007)^{10}$ has been indicated by Grésillon (1994, pp. 195-202) and also by Pierazzo (2009, pp. 171-173): namely digital editions where instead of cryptic symbols (like the ones used for the edition of Flaubert's Hérodias [1991]) one can use functions and actions which more intuitively disentangle the intricacies of the source document. In the Austen Digital Edition's website, for example, users can read an underlying text covered by a superimposed new reading by passing the mouse over a colour-coded section in the same way one would have used a magnifying glass.

But for editors of digital (diplomatic) editions, the needs of users influence not only the publication side (the front-end or interface) but also the scholarly model that determines the transcription. As Alois Pichler correctly notes:

The aim of transcription has often been defined as "to represent the original manuscripts as correctly as possible". [...] Whom do we want to serve with our transcriptions? Philosophers? Grammarians? Or graphologists? What is "correct" will depend on the answer to this question (1995, p. 690).

In the case of the Austen Digital Edition, for instance, consideration of potential users has driven our treatment of abbreviations: while an expert reader will have no problem in understanding abbreviated words, other users will probably prefer the possibility of seeing all words expanded. It was therefore decided to expand the 
abbreviations, leaving to the user the option of switching these expansions on and off at their pleasure.

How far should we go in considering the needs of the Others? It depends: if it is a matter of simplifying the lives of readers by decrypting the idiosyncratic habits of textual scholarship, the answer should probably be: 'quite a long way'. On the other hand, if it is matter of considering the needs of possible future scholars in other disciplines and providing special markup for them, the temptation is to say: 'not far'. There is, in fact, a serious risk of wasting precious project time here: it is very difficult to guess other scholars' needs or principles which are, potentially, infinite - as infinite as the set of "facts" that can be derived from the document.

\subsection{The Nature of the Document}

The same kind of features present in different types of manuscripts may need to be treated differently. Let us consider two examples: a richly illuminated manuscript and a calligramme (a text in which the typeface, calligraphy or handwriting is arranged in a way that creates a visual image). In both cases the editor will need to decide whether to include precise information about the graphical components of the manuscript or just transcribe the text: the decision and the selection will reflect the purpose and intended use of the transcription, but it will probably also be influenced by the different purposes of the graphic insertions (on the one hand decorative, on the other organic to the text).

Draft manuscripts often require a lot of effort to decipher or order the textual content, where unconventional or unpredictable layouts witness the genetic process of such a document. In those cases an editor might wish to include accurate topological information in the transcription or to order the different revisions chronologically. For the Austen Digital Edition, for instance, the layout of the documents has been respected 
(within some limits): in the fair copies and in particular in the Juvenilia it was clear that Austen intended to produce manuscripts that mimicked printed books, and so elements of the layout have been considered as organic to the transmitted texts. Elsewhere, specifically in the working draft manuscripts, the layout was captured in a slightly different way and for a different reason: deletions, additions and substitutions have been preserved, marked and displayed as closely as possible to the original position and layout, in an attempt to extricate the minutiae of the revision process.

\subsection{The Capabilities of the Publishing Technology}

Even if it is true that a digital edition can represent many more features than a print publication can, we know that the web has some limitations: we cannot, for instance, reproduce easily some of the calligrammes mentioned before in HTML, the technology which dominates the web today. Even more problematic is the representation of inconsistent baselines or the variable size of graphs. If the display of a given feature cannot be achieved within the chosen technical infrastructure, then editors have several choices:

- Record the information in the source anyway: sooner or later the publishing technology will allow for it. ${ }^{11}$

- Avoid recording the information: it is pointless and time consuming if no output is to be expected.

- Select a different output technology. ${ }^{12}$

For instance, in the Austen Digital Edition the struggle has been to position the interlinear insertions where they were in the original manuscripts. The varying dimension and spacing of the handwriting made the attempt to reproduce it very difficult: every word has been isolated and moved according to its relative position using CSS-based coding. The attempts have not been entirely successful and sometimes a less precise approximation 
than the one that was hoped for had to be accepted, but it is hoped that what is recorded will suffice to help the reader to understand the author's revision habits. Furthermore, as the edition is to be presented side by side with the facsimile, the user is able to verify the exact documentary layout her- or himself.

\section{The Facsimile}

This reference to the facsimile leads to a further question. The inclusion or otherwise of a facsimile has been evoked in the preceding discussion as a discriminating factor in selecting the appropriate level of transcription: if a facsimile is included then it may reduce the need for details in the transcription. Are the parameters for selection given above therefore to be influenced by the presence of a facsimile in the publication website? Kevin Kiernan suggests they are, and indeed takes this idea still further: "the image-based scholarly edition subsumes the purpose of a diplomatic edition and removes the fruitless frustration of trying to preserve the exact layout, illumination, and physical appearance of a manuscript in printed form" (2006, p. 266). Michael Hunter seems to be of the same opinion: "those particularly interested in a writer's methods of composition always have the alternative of recourse to a photographic or digital reproduction. Such consultation is preferable to even the most elaborate type-facsimile" (2007, p. 75$)$. This is a position that requires some thought. It states that a diplomatic edition is a pointless product when you are able to access (and publish) the (digital) pictures of the source. This implies that a diplomatic edition is simply an imperfect attempt to reproduce the original document, while the image is a more perfect reproduction of it.

This implication can be discussed from two different points of view. First, as was argued earlier, a diplomatic edition is the result of an editor's interpretation, of which the type-facsimile layout is one possible embodiment. If a diplomatic edition is a non-objective, 
interpretative operation, then it follows that is also a scholarly activity and can be justified on these grounds, in the same way that a critical edition can, with both presenting the scholarly and computational analysis of the chosen textual phenomena. It is true that a diplomatic edition is not a surrogate for the manuscript when a facsimile is also present, but it is rather a set of functions and activities to be derived from the manuscript which challenge the editorial work and force a more total engagement of the editor with the source document. As Kathryn Sutherland notes:

In a non-facsimile printed scholarly edition the diplomatic transcription normally stands in for the manuscript, ensuring that it is not the manuscript that provides authority for the edition but the implied precision of the editor's translation. By contrast, in the image-based digital edition the editor is continually on trial, open to account and correction (Sutherland and Pierazzo, 2011).

The dialectic relationship between the diplomatic edition and the facsimile representation, while demanding extreme editorial rigour, engages the users in close inspection of the translations enacted by the editor in a sort of ideal, involving, competition.

The second criticism of the suggestion that facsimile editions supplant diplomatic ones arises from the very nature of the digital diplomatic edition, which is a composite, complex, scholarly-informed and at the same time transient object. The facsimile is a very poor surrogate for this, as we will soon discover.

\section{Documentary Digital Editions}

In the digital world, and especially for TEI users, we are now used to thinking of the data model (the source) and the publication (the output) as two separate objects. The typefacsimile or document layout is only one of the possible outputs of the source and perhaps not the most meaningful. In the Austen Digital Edition, for instance, users are allowed to modify the output by switching on and off abbreviations, line breaks, deletions, and additions; in this way the output ranges from a diplomatic to a reading view of the text. 
These possibilities offered by digital editions based on markup languages (and in particular those based on TEI) are not new or complicated at all. However, they do imply that we should redefine what we mean by diplomatic edition. According to the definition given at the beginning of this article, a diplomatic edition is a published version of a transcription which reproduces as many of the characteristics of the original document as the medium permits or as the project requires. On the other hand, an edition based on text encoding will normally be composed of many parts, including at a minimum the following:

- A source file (or files) which will contain the transcribed text. At the current time XML-TEI markup represents the best practice of text encoding in the Humanities, but other formats are (and will continue to be) possible as well. One of the reasons why the TEI model is particualry effective is because it enables the encoding and transcription of several alternatives for the same segment allowing, for example, the encoding of abbreviated forms along with the expanded ones, or of erroneous passages and their corrections.

- A set of scripts able to read the source file and transform it into some particular 'view' of the text. The scripts therefore express which combination of encoded features compose the particular views. These scripts will typically be XSLT or XQuery if the source file is XML.

- One or more outputs, each of which will represent one of the aforementioned views or, to use a more established word, one 'edition'. This may include a diplomatic view if the editors have decided that this is a purpose of the edition.

- One or more styling files to refine the display of the edition. For output in HTML, this role will typically be filled by some combination of CSS and JavaScript. 
So, where is the diplomatic edition? Let us suppose that the transcription is recorded as an XML source file: in this case the transcription file will not look like the original document in any meaningful way. The output displays the transcribed text as closely as possible to the original document, but it is the scripts that store the knowledge (the scholarship) of how to produce such an edition. One might use a culinary metaphor here: the source contains the ingredients, the scripts contain the recipe, and the output represents the cooked dish. This metaphor works, but only to a certain level of abstraction because while the source contains the ingredients to produce the edition, it can in many circumstances be considered an edition by itself: for a TEI expert, for instance, an XML source file will be even more eloquent that the output. This consideration undermines an otherwise tempting classification: having said at the beginning that transcription represents the private first stage of editorial work with the source manuscript, while the diplomatic edition represents its public (published) face, one could argue that this division of work is reflected in the source as transcription and the output as diplomatic edition. However, as we have just observed, the source file can be considered an edition by itself (especially when XML-TEI is used), and in fact the importance of published source files as scholarly products has been widely recognized (Bodard and Garcés, 2009). The question can be pushed even further: in the case of the Austen Digital Edition, the diplomatic output is interactive and can be modified by the users, meaning that diplomatic is only one of the possible, unstable, states of the output; we might therefore conclude that this is not a diplomatic edition after all. But perhaps one of the most important aspects of such practice is that, assuming that the text is encoded using the TEI Guidelines or using a very similar approach enabling multiple outputs, while the source file contains a diplomatic edition, it may also contains other editions - semi-diplomatic, reading, critical, interpretative, or others - all of which can be contained simultaneously in potentia within the same source file, 
with each of these editions requiring the application of different sets of scripts and styling to be realised. This proteiform, cumulative nature of digital editions challenges on the one hand the concept of a single edited text. Which is 'the text'? The redundant, paradigmatically encoded text or one of the many possible outputs? Similarly challenged on the other hand is the commonly accepted way of distinguishing different editorial products, namely the different levels of editorial intervention. The handling of features such as corrections of scribal errors, preservation of original spellings, layout and paginations are normally used to distinguish diplomatic from semi-diplomatic from reading editions, with each of them characterized by an increasing weight of editorial intervention. For digital editions based on text encoding the editorial interventions are all present at once in the source, they are just not displayed at once.

The picture becomes even more complex if we consider that scripts and stylesheets are also normally used to produce intermediate outputs. During the editorial process one often needs to produce different views of the text, such as tabular pre-publication views, list of words, etc. These may have the twofold use of helping the editor check the consistency and progress of the work, but also providing documentation of the editorial practices which so often are only briefly outlined in the introductory material. These documentary by-products of the editorial work have been defined as mesotext by Peter Boot, who has claimed them as a fundamental part of the editorial work in a digital framework (Boot, 2007). Scripts and stylesheets, then, far from being only the realm of the "techies", store, for instance, the knowledge of how to exploit the dynamism implied in the redundant, paradigmatic TEI markup. ${ }^{13}$ The role of programming within the system described above is therefore not to be underestimated, and neither is its implicit scholarly content. 
At a different level, styling scripts in a language like CSS (or indeed JavaScript) are also of capital importance in modern, best-practice HTML websites. Again the Austen Digital Edition can give an iconic demonstration of this affirmation: one has just to try to display any of the pages containing the diplomatic edition ${ }^{14}$ after disabling the CSS (a function available on most browsers), or to open it within a text-only browser such as Lynx, for instance, to understand that the more we try to find out what a diplomatic edition is in a digital environment, the more this concept becomes inclusive and therefore elusive.

Perhaps we should just stop trying to map digital editions to printed ones and instead recognize that we are producing a different type of object, one that we can perhaps call a documentary digital edition. This new object necessarily comprises all three components of a digital publication - the source, the output and the tools to produce and display it - and it is worth emphasizing again that all three are scholarly products that result from editorial practice.

The discussion of the features listed previously and the parameters for choosing the level of transcription that is appropriate to a specific project shows that the principal drive behind the decision-making process should be the purpose of the edition. Nevertheless, this argument leaves open the answer to questions like "Where to stop" or "Which features should I include". The discussion has demonstrated that one cannot declare once and for all which features should be included. In a famous article Tanselle listed the essential features that need to be retained in a diplomatic transcription of modern holographic documents (1978, pp. 50-51), but such lists are neither possible nor desirable for digital editions: in fact the editor's goal needs no longer be “to reproduce in print as many of the characteristics of the document as he can" $(1978$, p. 50) but rather to achieve the scholarly purpose of the transcription - a purpose which, by definition, varies. 
From the discussion it follows that we could define this new type of editorial object, the documentary digital edition, as the recording of as many features of the original document as are considered meaningful by the editors, displayed in all the ways the editors consider useful for the readers, including all the tools necessary to achieve such a purpose.

The discussion has also proved how a digital edition is something completely different with respect to a print-based one as it is made of different things, works differently, and implies different approaches to the text. In his Foreword to Electronic Textual Editing Tanselle declares "Printed and electronic renderings [sic!] are thus not ontologically different; they may be made of different physical materials, but the conceptual status of the text in each case is identical" (2006, p. 6). However this assertion is hard to sustain. When it comes to digital editing most of the print-oriented questions and the recommendations for good-practice that Tanselle outlined are not only not central but mostly not applicable in the new medium. Printed and digital editions may have the same function, namely to make a given text available to an audience, but the way they have to be prepared, the kind of questions the editor needs to answer, and ultimately their very natures are substantially, if not ontologically, different. 


\section{Notes}

1. It would be too long to list all the contributions to the debate, many of which are mentioned further below in the present essay. At this point I will therefore only refer to Deegan and Sutherland, 2009, for an overview.

2. This article both condenses and elaborates on two papers I have presented at Digital Humanities conferences, in 2006 at Université Paris-Sorbonne, Paris, and in 2009 at University of Maryland, College Park.

3. See the Jane Austen's Fiction Manuscripts Digital Edition website (http://www.janeausten.ac.uk) where the aims and the purposes of the edition are summarized by the Director, Professor Kathryn Sutherland, as follows: "The focus of the present digital edition is three-fold: the virtual reunification of this significant collection of fiction manuscripts by means of high-quality digital photographic images; the linking of these images to fully encoded and searchable diplomatic transcriptions; and the creation of as complete a record as possible of the conservation history and current physical state of these frail objects". I am grateful to Professor Sutherland for material from the edition used in the argument of this article.

4. For an historical overview on different types of edition see Greetham, 1994, pp. 347372.

5. "A single edition might contain both transcriptions and critical texts", (Meulen and Tanselle, 1999, p. 203).

6. 'A 'model' I take to be either a representation of something for purposes of study, or a design for realizing something new. [Models] are by nature simplified and therefore fictional or idealized representations" (McCarty, 2005, p. 24). The full chapter on Modelling 
(pp. 20-72) can be used as an introduction to the theory of models in a Digital Humanities context.

7. The main editorial responsibilities for the Austen Digital Edition reside with Professor Kathryn Sutherland. Other members of the team who contributed to the definition of the editorial model were: Jenny McAuley, Elena Pierazzo and Sharon Regaz.

8. See the website of the Working Group for Documentary and Genetic Encoding: http://www.tei-c.org/SIG/Manuscripts/genetic.html.

9. For the criticism of critical apparatus see again, among others, Lavagnino, 2009, pp. 6667.

10. For instance, speaking of square brackets, he notes that: "these have been used for almost diametrically opposite purposes - to indicate deletions in the original; to denote text lost through mutilations; or to denote editorial supply", pp. 118-20

11. See, for instance, how the introduction of CSS2 and CSS3 has already enhanced the expressive capability of HTML in a way that was not really imaginable just few years ago, as well as HTML 5 has pushed much forward the boundaries of the language itself. Therefore we can easily suppose that the limits of today will not be the same as those of tomorrow. 12. Macromedia Flash, for example, is able to overcome many of the limitations of HTML, but has other drawbacks, particularly with regard to openness and sustainability.

13. This view of TEI markup is in deliberate contrast to the one expressed by Jerome McGann, according to whom the TEI markup "freezes" the multiple function spaces of the text by giving them a "finite" determination (2004, p. 205).

14. See, for instance, page 1 of The Watsons for a dramatic effect: http://www.janeausten.ac.uk/manuscripts/pmwats/1.html 


\section{References}

Bodard, G., and Garcés, J. (2009). Open Source Critical Editions: A Rationale. In

Deegan M., and Sutherland K. (eds.), Text Editing, Print and the Digital World. Aldershot: Ashgate, pp. 83-98.

Boot, P. (2007). Mesotext. Framing and Exploring Annotations. In Stronks E., and Boot P. (eds.), Learned Love. Proceedings of the Emblem Project Utrecht Conference on Dutch Love Emblems and the Internet (November 2006). The Hague: DANS - Data Archiving and Networked Services, pp. 211-225.

Deegan, M. (2006). Collection and Preservation of Electronic Edtions. In Burnard L., O’Brien O'Keeffe K., and Unsworth J. (eds.), Electronic textual editing. New York: Modern Language Association of America, pp. 358-370.

Deegan M., and Sutherland K. (eds.) (2009). Text Editing, Print and the Digital World. Aldershot: Ashgate.

Driscoll, M. J. (2006). Levels of Transcription. In Burnard L., O'Brien O'Keeffe K. and Unsworth J. (eds.), Electronic textual editing. New York: Modern Language Association of America.

Elsschot, W. (2007). Achter de Schermen. Peter de Bruijn V. N., and Hulle D. V. (eds.). Center for Manuscript Genetics (Universiteit Antwerpen), Huygens Instituut.

Flaubert, G. (1991). Hérodias. Bonaccorso G. and collaborators (eds.). Paris: Nizet.

Gabler H. W., Bornstein G., and Pierce G. B. (eds.) (1995). Contemporary German editorial theory. Ann Arbor: University of Michigan Press.

Gabler, H. W. (2007). The Primacy of the Document in Editing. Ecdotica, 4: 197-207.

Greetham, D. C. (1994). Textual Scholarship: An Introduction. New York: Garland. 
Grésillon, A. (1994). Eléments de critique génétique: lire les manuscrits modernes. Paris: Presses universitaires de France.

Huitfeldt, C., and Sperberg-McQueen, C. M. (2008). What is transcription?. Literary and Linguistic Computing, 23: 295-310.

Huitfeldt, C. (2006). Philosopy Case Study. In Electronic textual editing. In Burnard L., O’Brien O'Keeffe K., and Unsworth J. (eds.), Electronic textual editing. New York: Modern Language Association of America, pp. 181-196.

Hunter, M. (2007). Editing Early Modern Texts: an introduction to principles and practice. New York: Palgrave Macmillan.

Kiernan, K. (2006). Digital Facsimile in Editing. In Electronic textual editing. In Burnard L., O’Brien O'Keeffe K., and Unsworth J. (eds.), Electronic textual editing. New York: Modern Language Association of America, pp. 262-268.

Lavagnino, J. (2009). Access. Literary and Linguistic Computing, 24: 63-76.

McCarty, W. (2005). Humanities Computing. Basingstoke: Palgrave Macmillan.

McGann, J. (2004). Marking Texts of Many Dimensions. In Schreibman S., Siemens R. and Unsworth J. (eds.), A Companion to Digital Humanities. Oxford: Blackwwell, pp. 198-217. McLouglin, T. (2010). Bridging the Gap. In Rehbein, M. and Ryder, S. (eds.)

Computerphilologie, vol. 10, pp. 37-54.

Meulen, D. L. V. , and Tanselle, G. T. (1999). A System of Manuscript Transcription. Studies in Bibliography, 52: 201-213.

TEI Consortium (ed.) (2009). TEI P5: Guidelines for Electronic Text Encoding and Interchange. 1.7.0. TEI Consortium. http://www.tei-c.org/Guidelines/P5/ (Accessed 6 August 2010). Pichler, A. (1995). Transcriptions, Texts and Interpretation. In Johannessen K., and Nordenstam T. (eds.), Culture and Value: Philosophy and the Cultural Sciences. Wien: Austrian Ludwig Wittgenstein Society, pp. 690-695. 
Pierazzo, E. (2009). Digital Genetic Editions: The Encoding of Time in Manuscript Transcription. In Deegan M., and Sutherland K. (eds.), Text Editing, Print and the Digital World. Aldershot: Ashgate, pp. 169-186.

Rehbein, M. (2010). The Transition from Classical to Digital Thinking. Reflections on Tim McLoughlin, James Barry and Collaborative Work. In Rehbein, M. and Ryder, S. (eds.) Computerphilologie, vol. 10, pp. 55-67.

Robinson, P. M. W., and Solopova, E. (1993). Guidelines for Transcription of the Manuscripts of the Wife of Bath's Prologue. In Blake N. F., and Robinson P. M. W. (eds.), The Canterbury Tales Project: Occasional Papers. Oxford University Computing Services, Office for Humanities Communication vol. 1, pp. 19-52.

Robinson, P. M.W. (2002). Where we are with Electronic Scholarly Editions, and where we want to be. Computerphilologie, 4. http://computerphilologie.tudarmstadt.de/jg03/robinson.html (accessed 6 August 2010).

Robinson, P. M.W. (2009). What text really is not, and why editors have to learn to swim. Literary and Linguistic Computing, 24: 41-52.

Rosenblueth, A., and Wiener, R. (1945). The Role of Models in Science. Philosophy of Science, 12: 316-322.

Sperberg-McQueen, C. M. (2009). How to teach your edition how to swim. Literary and Linguistic Computing, 24: 27-52.

Sutherland, K., and Pierazzo, E. (2011). The Author's Hand: from Page to Screen. In Deegan M., and McCarty W. (eds.), Collaborative Research in the Digital Humanities. Aldershot: Ashgate (forthcoming).

Tanselle, G. T. (1978). The Editing of Historical Documents. Studies in Bibliography, 31: 156. 
Tanselle, G. T. (2006). Foreword. In Burnard L., O’Brien O'Keeffe K., and Unsworth J. (eds.), Electronic textual editing. New York: Modern Language Association of America, pp. 16.

Vanhoutte, E. (2000). Where is the editor? Resistance in the Creation of an Electronic Critical Edition. In Deegan, M., Anderson, J., and Short, H. (eds.) DRH 98. Selected papers from Digital resources for the Humanities 1998 University of Glasgow, September 1998. Office for Humanities Communication, London, 171-183.

Zeller, H. (1995). Record and Interpretation: Analysis and Documentation as Goal and Method of Editing. In Gabler H. W. , Bornstein G., and Pierce G. B. (eds.) Contemporary German editorial theory. Ann Arbor: University of Michigan Press, pp.17-58. 